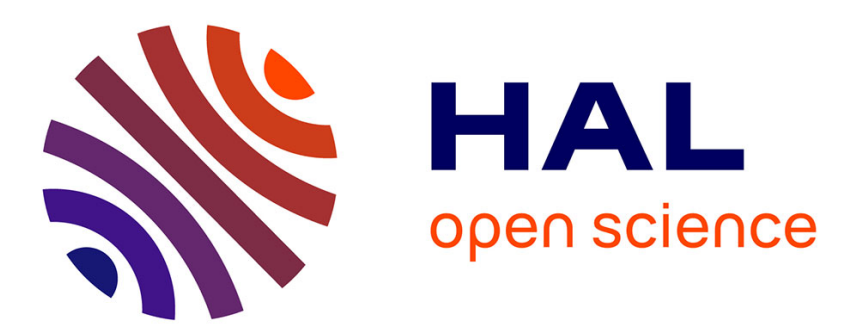

\title{
Reduced order model in cardiac electrophysiology with approximated Lax pairs
}

Jean-Frédéric Gerbeau, Damiano Lombardi, Elisa Schenone

\section{To cite this version:}

Jean-Frédéric Gerbeau, Damiano Lombardi, Elisa Schenone. Reduced order model in cardiac electrophysiology with approximated Lax pairs. Advances in Computational Mathematics, 2015, 41 (5), pp.1103-1130. 10.1007/s10444-014-9393-9 . hal-01093488v2

\section{HAL Id: hal-01093488 \\ https://hal.science/hal-01093488v2}

Submitted on 7 Dec 2015

HAL is a multi-disciplinary open access archive for the deposit and dissemination of scientific research documents, whether they are published or not. The documents may come from teaching and research institutions in France or abroad, or from public or private research centers.
L'archive ouverte pluridisciplinaire HAL, est destinée au dépôt et à la diffusion de documents scientifiques de niveau recherche, publiés ou non, émanant des établissements d'enseignement et de recherche français ou étrangers, des laboratoires publics ou privés. 


\title{
Reduced Order Model in Cardiac Electrophysiology with Approximated Lax Pairs
}

\author{
Jean-Frédéric Gerbeau* ${ }^{* \dagger}$, Damiano Lombardi*†, Elisa Schenone*
}

\begin{abstract}
A reduced-order method based on Approximated Lax Pairs (ALP) is applied to the integration of electrophysiology models. These are often high-dimensional parametric equation systems, challenging from a model reduction standpoint. The method is tested on two and three dimensional test-cases, of increasing complexity. The solutions are compared to the ones obtained by a finite element. The reducedorder simulation of pseudo-electrocardiograms based on ALP is proposed in the last part.
\end{abstract}

\section{Introduction}

This work ${ }^{1}$ is devoted to application of a reduced-order method based on Approximated Lax Pairs (ALP, see [12]) to cardiac electrophysiology. The ability of this method to handle propagation phenomena was considered in [12]. In the present paper, we focus more specifically on its behavior for parametric problems.

From a Reduced-Order Modeling (ROM) standpoint, cardiac electrophysiology is a challenging context. Indeed, the systems describing the electrical activation of tissues are nonlinear, they exhibit front propagations, and they depend on many parameters. For example, the possible presence of infarcted regions requires a modification of the space dependence of some reaction parameters. Similarly, the possible initiation of ectopic stimulations yields source terms which can change in space and in time. Consequently, a very large space of parameters has to be visited in order to cover all the possible scenarios.

The ability to deal with parametric systems is one of the main issues in model reduction. We refer for instance to [19] for a discussion on affinely parametrized elliptic

*Inria Paris-Rocquencourt, B.P. 105 Domaine de Voluceau, 78153 Le Chesnay, France

†Sorbonne Universités UPMC Univ Paris 6, UMR 7598 Laboratoire Jacques-Louis Lions, 75005 Paris, France. e-mails: jean-frederic.gerbeau@inria.fr, damiano.lombardi@inria.fr, elisa.schenone@inria.fr

${ }^{1}$ The published version of this article is available: Reduced order model in cardiac electrophysiology with approximated Lax pairs, Advances in Computational Mathematics: Volume 41, Issue 5 (2015), Page $1103-1130$ 
partial differential equation systems. The standard methods of model reduction are often based on the construction of a database of pre-computed simulations. Proper Orthogonal Decomposition is a paradigmatic example, which is widely used in many applications (see e.g. $[1,13,15,23])$. The need of a database can become an issue when dealing with high dimensional parametric spaces, since the construction of the database can be extremely demanding both in time and memory. The method ALP considered in this work does not rely on a database. It therefore avoids the need of an off-line exploration of a large parametric space. Instead, it makes a basis evolve in a way dependent on the dynamics of the solution. In all the examples given in this work, the method will be systematically compared to a full-order model obtained by finite element. For illustration purposes only, it will also be compared to the POD computed from a "simple" set of precomputed solutions.

The structure of the work is as follows. In Section 2 the equations of the cardiac electrophysiology are introduced. In Section 3, the ALP method is detailed. The general principle of the method is recalled in Section 3.1, useful complements are presented in Section 3.2, then the necessary steps to apply the method to the monodomain and bidomain equations are presented in Sections 3.3 and 3.4 respectively. In Section 4 various numerical experiments are proposed. The first example is a $2 \mathrm{D}$ propagation in an isotropic uniform medium. Then, a synthetic infarction is simulated and the problem of ectopic stimuli is investigated. The last case is the 3D simulation of a pseudo-electrocardiogram. Some perspectives of the present study are presented in the conclusion.

\section{Electrophysiology}

\subsection{The bidomain equations}

The electrical activity in the heart is modeled by the bidomain equations (see for instance $[18,21,25])$. At the microscopic level, the cardiac muscle is subdivided into an intracellular and an extracellular domain. The bidomain equations are obtained at the macroscopic level through a homogenization process which leads to the definition of an intra- and an extra-cellular electrical potential, denoted by $u_{I}$ and $u_{E}$ respectively. The bidomain equations read

$$
\begin{aligned}
A_{m}\left(C_{m} \frac{\partial v_{m}}{\partial t}+I_{i o n}\left(v_{m}, w\right)\right)-\operatorname{div}\left(\overline{\bar{\sigma}}_{I} \nabla v_{m}\right)-\operatorname{div}\left(\overline{\bar{\sigma}}_{I} \nabla u_{E}\right) & =A_{m} I_{a p p} \\
-\operatorname{div}\left(\left(\overline{\bar{\sigma}}_{I}+\overline{\bar{\sigma}}_{E}\right) \nabla u_{E}\right)-\operatorname{div}\left(\overline{\bar{\sigma}}_{I} \nabla v_{m}\right) & =0 \\
\frac{\partial w}{\partial t}-g\left(v_{m}, w\right) & =0
\end{aligned}
$$

where $v_{m}=u_{I}-u_{E}$ is the transmembrane potential, $w$ is a variable related to the ionic activity, $\overline{\bar{\sigma}}_{I, E}$ denotes the intra- (resp. extra-) cellular conductivity tensor (see for instance [24]), $A_{m}$ and $C_{m}$ are the ratio of membrane area per unit volume and the 
membrane capacitance per area respectively. Finally, the term $I_{i o n}$ denotes a nonlinear function representing the ionic current through the cell membrane and the term $I_{a p p}$ is a given source term. More details are given in the numerical experiments section.

The bidomain equations are coupled to a ionic model in order to define the current $I_{i o n}$. For simplicity, in this study we use the FitzHugh-Nagumo model $[11,17]$ defined by

$$
\begin{aligned}
I_{\text {ion }}(u, w) & =s u(u-a)(u-1)+w \\
g(u, w) & =\epsilon(\gamma u-w)
\end{aligned}
$$

where $0<a<1, s, \epsilon, \gamma$ are parameters. The FitzHugh-Nagumo model is only phenomenological and too crude to describe the current in cardiac cells. But it is sufficient to investigate our method in configurations close to what would be encountered in more realistic settings.

\subsection{The monodomain equations}

The bidomain equations are useful for some specific applications where the extracellular potential is needed. This is the case for example for electrocardiogram simulation (see e.g. [3] and Section 4.6 below) or to model complex electrophysiological behaviors like the virtual electrode phenomenon (see e.g. [9]). But in many cases, an approximation called "monodomain equations" proves to be sufficient [10]. These equations will be used in some of the numerical experiments presented below. They read:

$$
\begin{array}{r}
A_{m}\left(C_{m} \frac{\partial v_{m}}{\partial t}+I_{i o n}\left(v_{m}, w\right)\right)-\operatorname{div}\left(\overline{\bar{\sigma}}_{m} \nabla v_{m}\right)=A_{m} I_{a p p} \\
\frac{\partial w}{\partial t}-g\left(v_{m}, w\right)=0,
\end{array}
$$

where $\overline{\bar{\sigma}}_{m}$ is an electrical conductivity.

\section{The ALP method in electrophysiology}

In this section the reduced-order method used in the present work is detailed. It is hereafter derived for a generic PDE of the form:

$$
\partial_{t} u=F\left(u, \partial_{x}^{(n)} u\right)
$$

where $u(x, t), \quad x \in \Omega \subseteq \mathbb{R}^{d}, t \in(0,1)$ is the solution of the PDE, with specified initial and boundary conditions. For simplicity, $F\left(u, \partial_{x}^{(n)} u\right)$ will be denoted by $F(u)$.

The basic idea of the method, first proposed in [12], is to define a time evolving modal expansion of the form:

$$
\hat{u}(x, t)=\sum_{i=1}^{N_{M}} \beta_{i}(t) \varphi_{i}(x, t),
$$


where $\hat{u}$ is the low dimensional approximation of the PDE solution, $\beta_{i}(t)$ is the representation of $\hat{u}$ in the space defined by the $N_{M}$ modes $\varphi_{i}(x, t)$. In order to completely define the basis evolution, an equation and an initial condition for the modes have to be specified. Other works in the literature deal with this type of decomposition: recent examples are the dynamic low-rank decomposition (see for instance [14]), the orthogonal field equations (see [22]) and the bi-orthogonal decomposition method presented in $[7,8]$ to integrate stochastic PDEs.

The hypothesis and the governing equations of the ALP method are detailed in the remainder of this section. It will be assumed that $\left\langle\varphi_{i}, \varphi_{j}\right\rangle=\delta_{i j}, \forall t$, where $\langle\cdot, \cdot\rangle$ is the standard $L^{2}(\Omega)$ scalar product, $\delta_{i j}$ is the Kronecker delta.

Among all the possible formalisms that allow us to define a time-travelling mode expansion, an operator-based approach is chosen. The modes are retrieved in the set of the eigenfunctions of a time varying operator $\mathcal{L}_{\chi}$ which linearly depends upon the solution of the partial differential equation. The operator is represented by its spectrum and its eigenfunctions, which evolve under the action of an evolution operator (the same for all the eigenfunctions), denoted by $\mathcal{M}$. When applied to integrable systems of equations, these operators are the representation of a Lax pair, as pioneered in [16].

\subsection{The ALP method}

In this section, we gather the main results presented in [12]. The modes are, at every time instant, a subset of the eigenfunctions of a linear operator of Schrödinger-type:

$$
\mathcal{L}_{\chi}(u) \varphi:=L \varphi-\chi u(x, t) \varphi
$$

where $\chi$ is a real scalar parameter and $L$ is typically $-\Delta$, or any other linear selfadjoint elliptic operator. Then, the modes $\varphi_{i}$ are defined as the eigenfunctions of $\mathcal{L}_{\chi}(u)$ :

$$
\mathcal{L}_{\chi}(u) \varphi_{i}=\lambda_{i} \varphi_{i}
$$

The choice of the operator is arbitrary (to some extent), and it will be commented in the last part of this work. Let us assume that $u$ has the regularity that justifies the following steps of the derivation.

The operator evolves in time since it depends upon the solution of the PDE. This has important consequences for parametric systems, as it will be investigated by means of numerical experiments. Moreover, it is linear, selfadjoint with a compact inverse for every time, i.e. it defines, for every time, an orthonormal basis which is a complete basis of $L^{2}(\Omega)$.

The basis evolution may be derived by taking the time derivative of the mode definition (7):

$$
\left(\mathcal{L}_{\chi}-\lambda_{i} \mathcal{I}\right) \partial_{t} \varphi_{i}=\dot{\lambda}_{i} \varphi_{i}+\chi F \varphi_{i}
$$


This equation defines the modes evolution. Remark that the PDE expression enters explicitly in the modes evolution. Since the whole modal set is a complete basis, this equation provides a way to compute a smooth change of basis and the representation of this change may be represented on the basis itself, at each time. Let us write:

$$
\partial_{t} \varphi_{i}=\mathcal{M}(u) \varphi_{i}
$$

The following proposition shows that it is possible to compute an approximate representation of $\mathcal{M}(u)$ and to derive an evolution equation satisfied by the eigenvalues of $\mathcal{L}_{\chi}(u)$.

Proposition 1 ([12]). Let $u$ be a solution of equation (4). Let $\mathcal{L}_{\chi}(u)$ be defined by $(7)$. Let $N_{M} \in \mathbb{N}^{*}$. For $m \in\left\{1, \ldots, N_{M}\right\}$, let $\lambda_{m}(t)$ be an eigenvalue of $\mathcal{L}_{\chi}(u(x, t))$, and $\varphi_{m}(x, t)$ an associated eigenfunction, normalized in $L^{2}(\Omega)$. Let $\mathcal{M}(u)$ be the operator defined in (9). Then the evolution of $\lambda_{m}$ is governed by

$$
\partial_{t} \lambda_{m}=-\chi\left\langle F(u) \varphi_{m}, \varphi_{m}\right\rangle,
$$

and the evolution of $\varphi_{m}$ satisfies, for $p \in\left\{1, \ldots, N_{M}\right\}$,

$$
\left\langle\partial_{t} \varphi_{m}, \varphi_{p}\right\rangle=M_{m p}(u)
$$

with

$$
\left\{\begin{array}{l}
M_{m p}(u)=\frac{\chi}{\lambda_{p}-\lambda_{m}}\left\langle F(u) \varphi_{m}, \varphi_{p}\right\rangle, \quad \text { if } p \neq m \text { and } \lambda_{p} \neq \lambda_{m} \\
M_{m p}(u)=0, \quad \text { if } p=m \text { or } \lambda_{p}=\lambda_{m} .
\end{array}\right.
$$

We will denote by $M(u) \in \mathbb{R}^{N_{M} \times N_{M}}$ the skew-symmetric matrix whose entries are defined by $M_{m p}(u)$.

The proof of this proposition is based on a direct computation and it is shown in detail in [12]. The matrix $M$ is a representation of the operator $\mathcal{M}$ on the modes at time $t$. This representation is convenient from a computational standpoint since it can easily be obtained from the expression $F(u)$ defining the PDE (4), without any a priori knowledge of $\mathcal{M}(u)$. With this approximation of $\mathcal{M}(u)$, the evolution of the modes can be computed according to the nonlinear dynamics of the system. This is an important difference with standard reduced-order methods, like POD, where the modes are fixed once for all. To set up a reduced order integration method, only a small number $N_{M}$ of modes will be retained.

Equation (7) defines a Hilbert basis, which is used to approximate the solution $u \in$ $L^{2}(\Omega)$ :

$$
\tilde{u}(x)=\sum_{m=1}^{N_{M}} \beta_{m} \varphi_{m}(x)
$$


Proposition 1 gives an approximated way to propagate the eigenmodes and the eigenvalues. Function $F(u)$ is approximated by $\tilde{F}(u)=\sum_{m=1}^{N_{M}} \gamma_{m} \varphi_{m}$. Using these approximations in the PDE (4), the following holds:

$$
\sum_{m=1}^{N_{M}} \dot{\beta}_{m} \varphi_{m}+\beta_{m} \partial_{t} \varphi_{m}=\sum_{m=1}^{N_{M}} \gamma_{m} \varphi_{m}
$$

Projecting this relation on $\varphi_{p}$, and using (11), the expression of the PDE on the reduced basis is obtained:

$$
\dot{\beta}+M \beta=\gamma
$$

Defining $\left.\Theta_{i j}=\left\langle\tilde{F}(u) \varphi_{j}, \varphi_{i}\right\rangle\right),(10)$ and (11) are approximated by

$$
\dot{\lambda}_{i}=-\chi \Theta_{i i}
$$

and, for $\lambda_{i} \neq \lambda_{j}$,

$$
M_{i j}=\frac{\chi}{\lambda_{j}-\lambda_{i}} \Theta_{i j}
$$

respectively. The third order tensor $\left\langle\varphi_{k} \varphi_{j}, \varphi_{i}\right\rangle$ is denoted by $T_{i j k}$. By definition:

$$
\Theta_{i j}=\left\langle\tilde{F}(u) \varphi_{j}, \varphi_{i}\right\rangle=\sum_{k=1}^{N_{M}} \gamma_{k} T_{i j k} .
$$

Computing the time derivative of $T_{i j k}$ gives:

$$
\dot{T}_{i j k}=\left\langle\partial_{t} \varphi_{k} \varphi_{j}, \varphi_{i}\right\rangle+\left\langle\varphi_{k} \partial_{t} \varphi_{j}, \varphi_{i}\right\rangle+\left\langle\varphi_{k} \varphi_{j}, \partial_{t} \varphi_{i}\right\rangle
$$

Thus

$$
\dot{T}_{i j k}=\{M, T\}_{i j k}^{(3)},
$$

where

$$
\{M, T\}_{i j k}^{(3)}=\sum_{l=1}^{N_{M}}\left(M_{l i} T_{l j k}+M_{l j} T_{i l k}+M_{l k} T_{i j l}\right) .
$$

For the specific problem of interest, a relation linking $\gamma$ and $\beta$ will be also derived.

The set of equations which describes the dynamics in the reduced order space is:

$$
\left\{\begin{aligned}
\dot{\beta}_{i}+\sum_{m=1}^{N_{M}} M_{i m} \beta_{m}-\gamma_{i} & =0 \\
\dot{\lambda}_{i}+\chi \sum_{m=1}^{N_{M}} T_{i i m} \gamma_{m} & =0 \\
\dot{T}_{i j k} & =\{M, T\}_{i j k}^{(3)}, \\
M_{i j} & =\frac{\chi}{\lambda_{j}-\lambda_{i}} \sum_{m=1}^{N_{M}} T_{i j m} \gamma_{m}, \\
\gamma_{i} & =\gamma_{i}(\beta),
\end{aligned}\right.
$$


for $i, j, k=1 \ldots N_{M}$.

Remark. The complexity of the resolution of system (15) scales with $N_{M}^{3}$. As a consequence, the method is only efficient when a small number of basis functions is sufficient to reach the desired accuracy. This actually happens in many cases, as shown in Section 4.3. For the cases when a higher number of modes is required, a less expensive variant of the method is currently under investigation.

\subsection{Complements on the ALP method}

\subsubsection{Evolution of more complex tensors}

In the previous section, it was shown how the third order tensor $T_{i j k}$ evolves in time. Depending on the partial differential equation of interest, other tensors may appear in equation $(15)_{5}$. For the electrophysiology problems considered in this work, it will be useful to study the following type of tensor:

$$
\mathcal{A}_{i_{1}, \ldots, i_{k}}:=\int_{\Omega} \ell_{1}\left(\varphi_{1}\right) \ldots \ell_{k}\left(\varphi_{k}\right) d \Omega
$$

where the $\ell_{i}$ are linear operators which commute with time. The time evolution equation for this tensor reads:

$$
\partial_{t} \mathcal{A}_{i_{1}, \ldots, i_{k}}=\sum_{h=1}^{k} \int_{\Omega} \ell_{i}\left(\partial_{t} \varphi_{h}\right) \ldots \ell_{k}\left(\varphi_{k}\right) d \Omega=\sum_{h=1}^{k} \sum_{l=1}^{N_{M}} M_{h l} \mathcal{A}_{i_{1}, \ldots, i_{l}, \ldots, i_{k}} .
$$

Let us consider an example with a fourth-order tensor:

$$
D_{i j k h}=\int_{\Omega} \ell_{1}\left(\varphi_{i} \varphi_{j}\right) \ell_{2}\left(\varphi_{k} \varphi_{h}\right) d \Omega
$$

Then, differentiating in time

$$
\partial_{t} D_{i j k h}=\{M, D\}_{i j k h}^{(4)}=\sum_{l=1}^{N_{M}} M_{i l} D_{l j k h}+\sum_{l=1}^{N_{M}} M_{j l} D_{i l k h}+\sum_{l=1}^{N_{M}} M_{k l} D_{i j l h}+\sum_{l=1}^{N_{M}} M_{h l} D_{i j k l} .
$$

This will be used in Section 3.3.

\subsubsection{Reconstruction in the high dimensional space}

The reconstruction of the solution in the high dimensional space is now detailed. Contrary to standard approaches, this step is not trivial since the basis is time-evolving. 
The derivative of the modes can be approximated in the space defined by the modes at the current time instant as follows:

$$
\partial_{t} \varphi_{i} \approx \sum_{h=1}^{N_{M}} M_{h i} \varphi_{h}
$$

where the matrices $M \in \mathbb{R}^{N_{M} \times N_{M}}$ have been computed during the integration stage.

The practical implementation of the algorithm is the following. The modes are extracted at the very beginning by solving the spectral problem. The initial space is $\Phi^{(0)}:=\operatorname{span}\left\{\varphi_{1}(x, 0), \ldots, \varphi_{N_{M}}(x, 0)\right\}$.

Then, at every time instant a two-step scheme is applied. First, the modal space $\Phi$ is updated:

$$
\Phi^{(n+1)}=\Phi^{(n)}+\Delta t \mathcal{H}\left(M, \partial_{t} \Phi\right),
$$

where $\Phi \in \mathbb{R}^{\mathcal{N} \times N_{M}}$ and $\mathcal{H}$ denotes an integration scheme (that can be either explicit or implicit).

For instance, if a second order Crank-Nicolson scheme is used, the update reads:

$$
\Phi^{(n+1)}=\Phi^{(n)}+\frac{\Delta t}{2}\left(\Phi^{(n+1)} M^{(n+1)}+\Phi^{(n)} M^{(n)}\right) .
$$

In general the scheme does not guarantee that the eigenfunctions at time $t^{(n+1)}$ are orthonormal. Hence, in a second step, a Modified Gram-Schmidt orthogonalization is applied to the space $\Phi$. The cost of the operation scales as $2 \mathcal{N} \times\left(N_{M}\right)^{2}$ and therefore is linear in the high order dimension $\mathcal{N}$. In this work this simple reconstruction was adopted and proved to be sufficiently precise.

Remark. For a better accuracy, the following alternative procedure could be used. An orthogonal complement can be added to the approximation of the time derivative:

$$
\partial_{t} \varphi_{i}=\sum_{h=1}^{N_{M}} M_{h i} \varphi_{h}+r_{i}
$$

where $\left\langle r_{i}, \phi\right\rangle=0$. To compute it, the expression of the derivative is injected into the equation governing the modes dynamics and projected on a basis orthogonal to the modes. Let us introduce $\Psi=\operatorname{span}\left\{\psi_{1}, \ldots, \psi_{N_{k}}\right\}$ such that $\Psi \perp \Phi$. At initial time the orthogonal basis is chosen as $\Psi=\operatorname{span}\left\{\varphi_{N_{M}+1}, \ldots, \varphi_{N_{M}+N_{k}}\right\}$. Then:

$$
\sum_{h=1}^{N_{M}} M_{h i}\left(\mathcal{L}_{\chi}-\lambda_{i} \mathcal{I}\right) \varphi_{h}+\left(\mathcal{L}_{\chi}-\lambda_{i} \mathcal{I}\right) r_{i}=\dot{\lambda}_{i} \varphi_{i}+F \varphi_{i}
$$

The linear system determining $r_{i}$ is obtained by projecting the equation onto $\Psi$ :

$$
\left\langle\left(\mathcal{L}_{\chi}-\lambda_{i} \mathcal{I}\right) r_{i}, \psi_{j}\right\rangle=\left\langle F \varphi_{i}, \psi_{j}\right\rangle .
$$


When this correction is taken into account, the cost of the method is higher. Indeed, at each time step, the set of $[\Phi, \Psi]$ have to be orthonormalized with the MGS method, leading to a cost proportional to $2 \mathcal{N} \times\left(N_{M}+N_{k}\right)^{2}$. For the test cases of the present work, this variant did not significantly improve the results.

\subsection{ALP for the monodomain equations}

We are now interested in applying the ALP method to the electrophysiology equations. We first derive the ALP algorithm for the monodomain equations (3). In order to apply the technique described above we couple (3) to the FitzHugh-Nagumo ionic model and write it as

$$
\begin{aligned}
A_{m} C_{m} \partial_{t} v_{m} & =f\left(v_{m}, w\right), \\
\partial_{t} w & =g\left(v_{m}, w\right),
\end{aligned}
$$

where

$$
f\left(v_{m}, w\right)=A_{m} s v_{m}\left(v_{m}-a\right)\left(1-v_{m}\right)-A_{m} w+\operatorname{div}\left(\overline{\bar{\sigma}}_{m} \nabla v_{m}\right)+A_{m} I_{a p p},
$$

and

$$
g\left(v_{m}, w\right)=\epsilon\left(\gamma v_{m}-w\right) .
$$

The first step to apply the ALP method is to define a linear operator $\mathcal{L}_{\chi}$. For the present work, the operator $\mathcal{L}_{\chi}$ is defined as

$$
\mathcal{L}_{\chi}(u) \varphi=-\operatorname{div}\left(\overline{\bar{\sigma}}_{m} \nabla \varphi\right)-\chi u \varphi
$$

and the modes $\varphi_{i}$ are solutions of the eigenproblem

$$
\mathcal{L}_{\chi}(u) \varphi_{i}=\lambda_{i} \varphi_{i}
$$

Let us remark that the conductivity $\overline{\bar{\sigma}}_{m}$ being a tensor, the modes set can account for some physical anisotropy due to the presence of cardiac fibers.

The eigenproblem is symmetric positive definite. In the examples presented below it is solved using a Krylov-Schur method with a Lanczos decomposition (resp. B-Lanczos for the generalized eigenproblem). Although only $N_{M}$ modes have to be computed, this step may be expensive. But it is worth noticing that it is solved only once, before the resolution of the reduced order model. In addition, the initial basis does not depend on the PDE parameters (except the conductivity tensor $\overline{\bar{\sigma}}_{m}$ ). Thus, the same initial basis can be used for different sets of parameters.

Let us now approximate the solution of equations (26) in the low dimensional space defined by the $N_{M}$ modes $\varphi_{i}(x, t)$ which are the first $N_{M}$ eigenfunctions defined by (30). The transmembrane potential $v_{m}$ can be approximated by

$$
\hat{v}_{m}=\sum_{i=1}^{N_{M}} \beta_{i}(t) \varphi_{i}(x, t) .
$$


We choose to approximate the ionic variable in the same reduced order space and write

$$
\hat{w}=\sum_{i=1}^{N_{M}} \mu_{i}(t) \varphi_{i}(x, t)
$$

where $\hat{w}$ is the low dimensional approximation of $w, \mu_{i}(t)$ is the representation of $\hat{w}$ in the reduced order space $\Phi$.

Using these Galerkin approximations, we can write:

$$
\begin{array}{r}
f\left(v_{m}, w\right) \approx-A_{m} s a \sum_{i=1}^{N_{M}} \beta_{i} \varphi_{i}+A_{m} s(a+1) \sum_{i, j=1}^{N_{M}} \beta_{i} \beta_{j} \varphi_{i} \varphi_{j}-A_{m} s \sum_{i, j, k=1}^{N_{M}} \beta_{i} \beta_{j} \beta_{k} \varphi_{i} \varphi_{j} \varphi_{k}+ \\
-A_{m} \sum_{i=1}^{N_{M}} \mu_{i} \varphi_{i}+\sum_{i=1}^{N_{M}} \beta_{i} \operatorname{div}\left(\overline{\bar{\sigma}}_{m} \nabla \varphi_{i}\right)+A_{m} I_{a p p}(x, t) .
\end{array}
$$

Using the approximation of $v_{m}$ in the reduced space $\Phi$, from equations (29) we find

$$
-\operatorname{div}\left(\overline{\bar{\sigma}}_{m} \nabla \varphi_{i}\right)-\chi \sum_{j=1}^{N_{M}} \beta_{j} \varphi_{j} \varphi_{i}=\lambda_{i} \varphi_{i}, \forall i=1, \ldots, N_{M}
$$

Let us substitute the first term of (34) in (33). Then the approximation of $f\left(v_{m}, w\right)$ writes

$$
\begin{array}{r}
f \approx-A_{m} s a \sum_{i=1}^{N_{M}} \beta_{i} \varphi_{i}-\sum_{i=1}^{N_{M}} \lambda_{i} \beta_{i} \varphi_{i}+A_{m} s(a+1) \sum_{i, j=1}^{N_{M}} \beta_{i} \beta_{j} \varphi_{i} \varphi_{j}-\chi \sum_{i, j=1}^{N_{M}} \beta_{i} \beta_{j} \varphi_{i} \varphi_{j}+ \\
\quad-A_{m} s \sum_{i, j, k=1}^{N_{M}} \beta_{i} \beta_{j} \beta_{k} \varphi_{i} \varphi_{j} \varphi_{k}-A_{m} \sum_{i=1}^{N_{M}} \mu_{i} \varphi_{i}+A_{m} I_{a p p}(x, t) .
\end{array}
$$

We can now project expression (35) onto $\Phi$. Then we define the projection of $f, \gamma_{p}$ $\forall p=1, \ldots, N_{M}$, as

$$
\begin{array}{r}
\gamma_{p}:=-A_{m} a \sum_{i=1}^{N_{M}} \beta_{i}\left\langle s \varphi_{i}, \varphi_{p}\right\rangle-\sum_{i=1}^{N_{M}} \lambda_{i} \beta_{i}\left\langle\varphi_{i}, \varphi_{p}\right\rangle-A_{m} \sum_{i=1}^{N_{M}} \mu_{i}\left\langle\varphi_{i}, \varphi_{p}\right\rangle+ \\
+A_{m}(a+1) \sum_{i, j=1}^{N_{M}} \beta_{i} \beta_{j}\left\langle s \varphi_{i} \varphi_{j}, \varphi_{p}\right\rangle-\chi \sum_{i, j=1}^{N_{M}} \beta_{i} \beta_{j}\left\langle\varphi_{i} \varphi_{j}, \varphi_{p}\right\rangle+ \\
-A_{m} \sum_{i, j, k=1}^{N_{M}} \beta_{i} \beta_{j} \beta_{k}\left\langle s \varphi_{i} \varphi_{j}, \varphi_{k} \varphi_{p}\right\rangle+A_{m}\left\langle I_{a p p}, \varphi_{p}\right\rangle .
\end{array}
$$


Let us assume that the applied stimulus is approximated by

$$
I_{a p p}(x, t)=\sum_{l=1}^{L} h_{l}(t) z_{l}(x) .
$$

If we use the orthonormality of $\Phi$, equation (36) can be written as

$$
\begin{aligned}
\gamma_{p}= & -\lambda_{p} \beta_{p}-A_{m} \mu_{p}-A_{m} a \sum_{i=1}^{N_{M}} B_{i p} \beta_{p}+A_{m}(a+1) \sum_{i, j=1}^{N_{M}} \beta_{i} \beta_{j} W_{i j p}+ \\
& -\chi \sum_{i, j=1}^{N_{M}} \beta_{i} \beta_{j} T_{i j p}-A_{m} \sum_{i, j, k=1}^{N_{M}} \beta_{i} \beta_{j} \beta_{k} Y_{i j k p}+A_{m} \sum_{l=1}^{L} h_{l}(t)\left\langle z_{l}, \varphi_{p}\right\rangle,
\end{aligned}
$$

where we define the matrix $B$, and the third and fourth order tensors $T, W, Y$ as

$$
\begin{array}{rll}
B_{i j}:=\left\langle s \varphi_{i}, \varphi_{j}\right\rangle & \forall i, j=1, \ldots, N_{M}, \\
T_{i j k}:=\left\langle\varphi_{i}, \zeta_{j k}\right\rangle & \forall i, j, k=1, \ldots, N_{M}, \\
W_{i j k} & :=\left\langle s \varphi_{i}, \zeta_{j k}\right\rangle & \forall i, j, k=1, \ldots, N_{M}, \\
Y_{i j k h}:=\left\langle s \zeta_{i j}, \zeta_{k h}\right\rangle & \forall i, j, k, h=1, \ldots, N_{M},
\end{array}
$$

and the functions $\zeta$ by

$$
\zeta_{i j}:=\varphi_{i} \varphi_{j} \quad \forall i, j=1, \ldots, N_{M} .
$$

Note that these tensors are computed once for all at the initialization phase. Then they are simply propagated according to their respective evolution equation.

Dealing with the second equation of (26), we write the projection of the approximated function $g\left(v_{m}, w\right)$ as

$$
\eta_{p}:=\left\langle g, \varphi_{p}\right\rangle=\epsilon\left(\gamma \beta_{p}-\mu_{p}\right), \quad \forall p=1, \ldots, N_{M} .
$$

Then, to solve equations (26) with the ALP method we have to compute at each time iteration the quantities

$$
\begin{aligned}
& \dot{\beta}_{i}+\sum_{j=1}^{N_{M}} \beta_{j} M_{j i}=\gamma_{i}, \\
& \dot{\mu}_{i}+\sum_{j=1}^{N_{M}} \mu_{j} M_{j i}=\eta_{i},
\end{aligned}
$$

where $\gamma_{i}, \eta_{i}$, for $i=1, \ldots, N_{M}$ are defined by (37) and (39). The complete set of equations that describe the dynamics is defined by (15)-(37)-(39), with equations (40) replacing the first equation of (15). 
Remark. Note that in the derivation of the ALP equations presented above, the diffusion term was eliminated in equation (35) by taking advantage of the modes definition in terms of the Schrödinger operator. Roughly speaking, a third order tensor is introduced, whose computation does not require modes differentiation. This is not the only possibility. Indeed, if the second term of (34) is substituted in (33), the approximation of $f\left(v_{m}, w\right)$ writes:

$$
\begin{array}{r}
f \approx-A_{m} s a \sum_{i=1}^{N_{M}} \beta_{i} \varphi_{i}-\frac{A_{m} s(a+1)}{\chi} \sum_{i=1}^{N_{M}} \lambda_{i} \beta_{i} \varphi_{i}-\frac{A_{m} s}{\chi^{2}} \sum_{i=1}^{N_{M}} \lambda_{i}^{2} \beta_{i} \varphi_{i}-A_{m} \sum_{i=1}^{N_{M}} \mu_{i} \varphi_{i}+ \\
-\frac{A_{m} s(a+1)}{\chi} \sum_{i=1}^{N_{M}} \beta_{i} \operatorname{div}\left(\overline{\bar{\sigma}}_{m} \nabla \varphi_{i}\right)-\frac{A_{m} s}{\chi^{2}} \sum_{i=1}^{N_{M}} \lambda_{i} \beta_{i} \operatorname{div}\left(\overline{\bar{\sigma}}_{m} \nabla \varphi_{i}\right)+\sum_{i=1}^{N_{M}} \beta_{i} \operatorname{div}\left(\overline{\bar{\sigma}}_{m} \nabla \varphi_{i}\right)+ \\
+\frac{A_{m} s}{\chi} \sum_{i, j=1}^{N_{M}} \beta_{i} \beta_{j} \operatorname{div}\left(\overline{\bar{\sigma}}_{m} \nabla \varphi_{i}\right) \varphi_{j}+A_{m} I_{a p p}(x, t) .
\end{array}
$$

Equation (41) is then projected onto $\Phi$. Let us make the same assumptions as below, i.e. separable form space-time sources. Then, the projection of $f, \gamma_{p} \forall p=1, \ldots, N_{M}$ is therefore defined as:

$$
\begin{aligned}
\gamma_{p}:=-A_{m} \mu_{p}-A_{m} \sum_{i=1}^{N_{M}}(a+ & \left.\frac{a+1}{\chi} \lambda_{i}+\frac{1}{\chi^{2}} \lambda_{i}^{2}\right) B_{i p} \beta_{i}+\sum_{i=1}^{N_{M}} A_{m}\left(\frac{a+1}{\chi}+\frac{1}{\chi^{2}} \lambda_{i}\right) \beta_{i} V_{i p}+ \\
& -\sum_{i=1}^{N_{M}} \beta_{i} E_{i p}-\frac{A_{m}}{\chi} \sum_{i, j=1}^{N_{M}} \beta_{i} \beta_{j} U_{i j p}+A_{m} \sum_{l=1}^{L} h_{l}(t)\left\langle z_{l}, \varphi_{p}\right\rangle,
\end{aligned}
$$

where we define the matrices $B, E, V$ and the third order tensors $U$ as

$$
\begin{array}{rll}
B_{i j} & :=\left\langle s \varphi_{i}, \varphi_{j}\right\rangle & \forall i, j=1, \ldots, N_{M}, \\
E_{i j}:=\left\langle\bar{\sigma}_{m} \nabla \varphi_{i}, \nabla \varphi_{j}\right\rangle & \forall i, j=1, \ldots, N_{M}, \\
V_{i j}:=\left\langle s \overline{\bar{\sigma}}_{m} \nabla \varphi_{i}, \nabla \varphi_{j}\right\rangle & \forall i, j=1, \ldots, N_{M}, \\
U_{i j k} & :=\left\langle\overline{\bar{\sigma}}_{m} \nabla \varphi_{i}, \nabla\left(\zeta_{j k}\right)\right\rangle & \forall i, j, k=1, \ldots, N_{M} .
\end{array}
$$

This way to proceed is attractive from a reduced-order modeling standpoint, since it allows to get rid of the fourth-order projection tensor appearing when the Galerkin projection of the FitzHugh-Nagumo ionic current is considered. For standard ROM based on Galerkin projection the scalability is of the order of $N_{M}^{4}$, while for ALP, by taking advantage of the definition of the potential in the Schrödinger operator, the cost of the ROM is of the order of $N_{M}^{3}$. A discussion on the cost and scalability of other ROM approaches is provided in $[5,20]$. An effective method to deal with nonpolynomial nonlinearities and to avoid the computation of high-order projection tensors could be inspired by the concept of empirical interpolation methods $[2,6]$. A comparison and an application of these methods to ALP will be the object of future works. 


\subsection{ALP for the bidomain equations}

To discretize the bidomain equations in the low dimension space $\Phi$ and apply the ALP method, as in section 3.3, we first choose the initial reduced order space. The operator $\mathcal{L}_{\chi}$ defined for this problem is given by

$$
\mathcal{L}_{\chi}(u) \varphi=-\operatorname{div}\left(\overline{\bar{\sigma}}_{I} \nabla \varphi\right)-\chi u \varphi
$$

where $\overline{\bar{\sigma}}_{I}$ is the intracellular conductivity tensor, indicated in the following as $\sigma_{I}$.

The second step is the discretization of equations (1). We can write the system as

$$
\begin{aligned}
A_{m} C_{m} \partial_{t} v_{m} & =f\left(v_{m}, u_{E}, w\right), \\
\partial_{t} w & =g\left(v_{m}, w\right), \\
q\left(v_{m}, u_{E}\right) & =0
\end{aligned}
$$

where

$$
\begin{aligned}
f\left(v_{m}, u_{E}, w\right) & =A_{m} s v_{m}\left(v_{m}-a\right)\left(1-v_{m}\right)-A_{m} w+\operatorname{div}\left(\sigma_{I} \nabla v_{m}\right)+\operatorname{div}\left(\sigma_{I} \nabla u_{E}\right)+A_{m} I_{a p p}, \\
g\left(v_{m}, w\right) & =\epsilon\left(\gamma v_{m}-w\right), \\
q\left(v_{m}, u_{E}\right) & =-\operatorname{div}\left(\left(\sigma_{I}+\sigma_{E}\right) \nabla u_{E}\right)-\operatorname{div}\left(\sigma_{I} \nabla v_{m}\right) .
\end{aligned}
$$

The projection onto the space $\Phi$ of the first equation of (46) gives

$$
\begin{aligned}
\gamma_{p}:=-\lambda_{p} \beta_{p}-A_{m} \mu_{p}-\lambda_{p} \xi_{p}-A_{m} a \sum_{i=1}^{N_{M}} \beta_{j} B_{i j}+A_{m}(a+1) \sum_{i, j=1}^{N_{M}} \beta_{i} \beta_{j} W_{i j p}-\chi \sum_{i, j=1}^{N_{M}} \beta_{i} \beta_{j} T_{i j p}+ \\
-\chi \sum_{i, j=1}^{N_{M}} \beta_{i} \xi_{j} T_{i j p}-A_{m} \sum_{i, j, k=1}^{N_{M}} \beta_{i} \beta_{j} \beta_{k} Y_{i j k p}+A_{m} \sum_{l=1}^{L} h_{l}(t)\left\langle z_{l}, \varphi_{p}\right\rangle, \quad \forall p=1, \ldots, N_{M},
\end{aligned}
$$

and the projection of function $g$ gives

$$
\eta_{p}:=\epsilon\left(\gamma \beta_{p}-\mu_{p}\right), \quad \forall p=1, \ldots, N_{M} .
$$

The projection of function $q$ gives a low dimensional linear system defined by

$$
\sum_{j=1}^{N_{M}} Q_{i j} \xi_{j}=-\sum_{j=1}^{N_{M}} E_{i j} \beta_{j}, \quad \forall j=1, \ldots, N_{M},
$$

where matrices $Q$ and $E$ are defined as

$$
\begin{array}{rlrl}
Q_{i j} & :=\left\langle\left(\sigma_{I}+\sigma_{E}\right) \nabla \varphi_{i}, \nabla \varphi_{j}\right\rangle & \forall i, j=1, \ldots, N_{M} \\
E_{i j}:=\left\langle\sigma_{I} \nabla \varphi_{i}, \nabla \varphi_{j}\right\rangle & \forall i, j=1, \ldots, N_{M} .
\end{array}
$$


To summarize, the set of equations which describes the dynamics of system (1) in the reduced order space is:

$$
\left\{\begin{aligned}
\dot{\beta}+M \beta-\gamma & =0 \\
\dot{\mu}+M \mu-\eta & =0 \\
\dot{\lambda}_{i}+\chi \sum_{m=1}^{N_{M}} T_{i i m} \gamma_{m} & =0, \quad i=1 \ldots N_{M} \\
\dot{T} & =\{M, T\}^{(3)}, \\
\dot{Y} & =\{M, Y\}^{(4)}, \\
\dot{Q} & =[M, Q], \\
\dot{E} & =[M, E], \\
M_{i j} & =\frac{\chi}{\lambda_{j}-\lambda_{i}} \sum_{m=1}^{N_{M}} T_{i j m} \gamma_{m}, \quad i, j=1 \ldots N_{M} \\
\gamma & =\gamma(\beta, \xi, \mu), \\
\eta & =\eta(\beta, \mu), \\
Q \xi & =-E \beta .
\end{aligned}\right.
$$

\section{Numerical experiments}

This section is devoted to numerical experiments. First, we consider the propagation of an electrical signal in a homogeneous tissue on a $2 \mathrm{D}$ domain. Then, some examples of spatial and temporal heterogeneity in parameters and source terms are proposed. The last application deals with pseudo-electrocardiograms and involve the bidomain equations coupled to a diffusion problem on a 3D domain.

\subsection{Preliminary comments about POD}

In the following numerical simulations, our main objective is to compare ALP with the finite element method (FEM). In some cases, we also compare ALP with the Proper Orthogonal Decomposition (POD).

The POD method consists in defining an orthonormal basis that maximizes the $L^{2}$ representation of a database of given snapshots, i.e. solutions computed in an off-line stage. The approach proposed in [23] is used to generate the basis. Let us recall its main steps. Consider an approximation of the solution in the form

$$
u\left(x, t_{i}\right) \approx \sum_{j} \hat{a}_{j}(t) \varphi_{j}(x)
$$


where $\varphi_{j}$ is the $j$-th POD mode. The modes are such that $\left\langle\varphi_{i}, \varphi_{j}\right\rangle=\delta_{i j}$ and:

$$
\varphi_{i}=\arg \min _{\tilde{\varphi}} \int_{0}^{T} \int_{\Omega}\left(u(x, t)-\sum_{j} \hat{a}_{j}(t) \varphi_{j}(x)\right)^{2} d \Omega d t .
$$

Let $u_{i}(x)$ be a snapshot of the database of pre-computed solutions. The minimization problem is solved by:

$$
\begin{array}{r}
A_{i j}:=\left\langle u_{i}, u_{j}\right\rangle, \\
A B=B S, \\
\varphi_{i}:=\frac{\sum_{j} B_{j i} u_{j}}{s_{i}^{1 / 2}} .
\end{array}
$$

An important remark is in order. POD is a way to approximate a space spanned by off-line solutions, and the result strongly depends on this space. When the parameters of the problems vary, it is necessary to consider a large number of configurations in order to build an off-line space as rich as possible. In electrophysiology, this strategy was carried out for example in [4]. In the present work, we only consider a very simple set of precomputed solutions: the ones obtained with nominal values of parameters. Thus, our POD results correspond to a best case scenario when the nominal values are used to run the reduced-order model, but to a (possibly) very bad scenario when the parameters are modified. In the latter case, it would be clearly possible to improve the POD results by enlarging the off-line dataset, but this would require to explore a huge number of configurations in the off-line stage. Although possible, this was not done here since POD was not the main topic of this paper.

\subsection{A few comments on the choice of $\chi$.}

The ALP basis depends upon a real positive scalar parameter $\chi$, that influences the spectrum and the eigenfunctions of the operator $\mathcal{L}_{\chi}$. The spectrum of the operator is discrete (the domain is bounded), real, and it can be divided into two parts: one corresponding to negative eigenvalues and one to positive (or null) eigenvalues. The larger the value of $\chi$, the higher is the number of negative eigenvalues. The larger the value of $\chi$, the more peaky the eigenfunctions corresponding to negative eigenvalues are. Thus, this parameter can be viewed as a characteristic length for the modes corresponding to negative eigenvalues. Let us illustrate this with a 1D numerical test case.

The domain is $\Omega=[0,1]$, the potential is the Gaussian $y=\exp \left(-250(x-0.5)^{2}\right)$. A piecewise linear finite element discretization is adopted, with $\mathcal{N}=256$. In Figure 1(a) the number of negative eigenvalues is shown for several values of the parameter $\chi$. For $\chi=0$, there are no negative eigenvalues. The number of negative eigenvalues increases with $\chi$. In Figure 1(b) the relative $L^{2}$ error in the reconstruction of the potential is shown when 


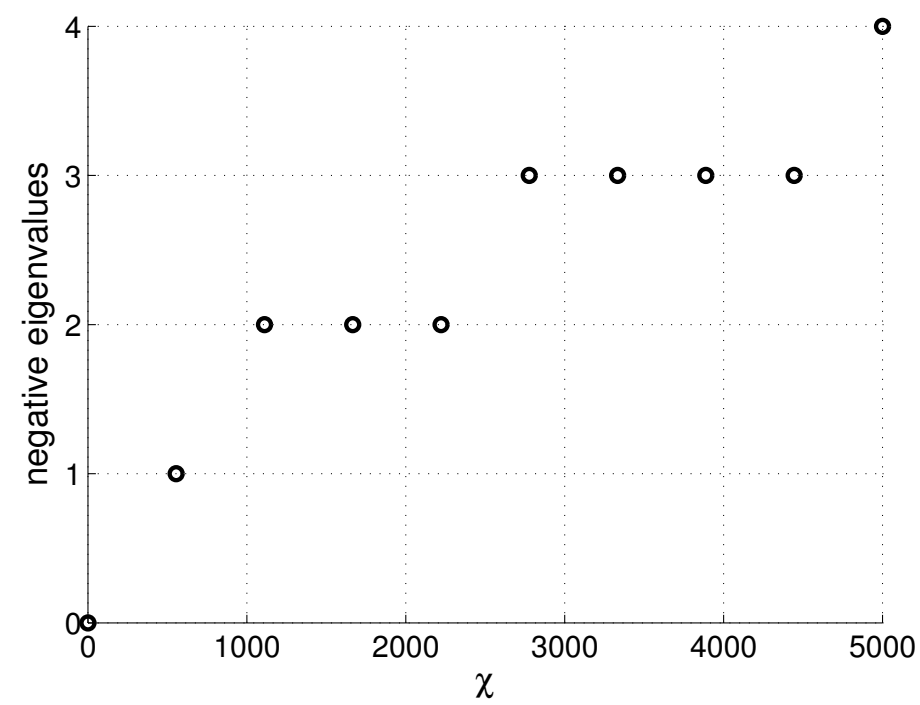

(a) Number of negative eigenvalues.

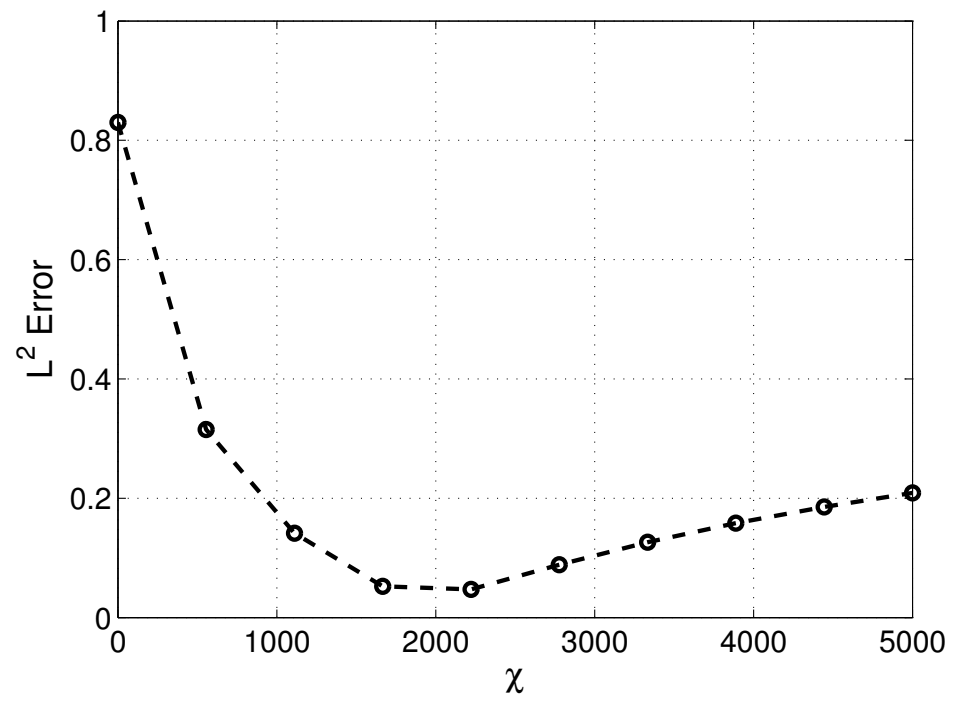

(b) Error in the reconstruction of the potential when the first mode only is used.

Figure 1: Properties when $\chi$ is varied. 
only the first mode is used, as a function of $\chi$. There is a minimum for $\chi \approx 2000$. This is related to the the fact that, for low values of $\chi$ the first mode has a characteristic length much higher than that of the gaussian potential, so that the reconstruction is poor. On the opposite, when $\chi$ is too large, the mode tends to a Dirac delta and it cannot represent the potential in a good way. The behavior of the first mode, when $\chi$ is varied, is shown in Fig. 2a.

Let us comment on the difference between the eigenfunctions corresponding to the negative spectrum and the eigenfunctions corresponding to the positive one. In Figure $2 \mathrm{~b}$ the first four modes are shown when $\chi=2000$. The first two modes correspond to the negative spectrum. Their shape is soliton-like: it is featured by a characteristic length and localized in space. The modes 3 and 4 correspond to the positive spectrum. They are sinus-like global modes, of increasing frequency, perturbed by the potential.

In practice, the value of $\chi$ is chosen after a preliminary numerical test, on a given setup, and depends on the domain size as well as on the $L^{2}$ norm of the potential. It is chosen in such a way that the initial datum is represented with a low relative error in the space spanned by few modes.

\subsection{Homogeneous parameters $2 \mathrm{D}$ case}

We consider a 2D square domain $[0,1]^{2}$, discretized with a P1 finite element mesh composed of 5.878 vertices, and the monodomain equations (3) with the parameters reported in Table 1. Concerning the ALP method, we solve the equations presented in Section 3.3. ALP results are compared with those obtained with the full order model (FEM) and the POD. The POD basis is generated by considering snapshots from the homogeneous 2D tissue propagation. For FEM simulations a second order Backward Difference method is used with time step $\delta t=0.1 \mathrm{~ms}$, while in the reduced order cases an Explicit Euler method is considered (time step $\delta t=0.01 \mathrm{~ms}$ ).

First we run a FEM simulation with the applied stimulus defined as

$$
I_{\text {app }}(\mathbf{x}, t)=h(t) z(\mathbf{x}),
$$

where $h$ is defined by

$$
h(t)=H(t)-H(t-5),
$$

$H$ denotes the Heaviside function, and $z$ is defined by

$$
z(\mathbf{x})=0.04 \cdot \mathbb{1}_{\Omega_{c}}, \Omega_{c}=\{(x, y) \text { s.t. }\|(x-0.25, y)\| \leq 0.5\} .
$$

\begin{tabular}{cccccccc}
\hline \hline$A_{m}^{2 \mathrm{D}}\left(\Omega \cdot \mathrm{mV}^{-1}\right)$ & $A_{m}^{3 \mathrm{D}}\left(\Omega \cdot \mathrm{mV}^{-1}\right)$ & $C_{m}\left(\mathrm{~mA} .{\mathrm{ms} . \mathrm{cm}^{-2}}^{2}\right.$ & $\sigma_{m}$ & $s$ & $a$ & $\epsilon$ & $\gamma$ \\
\hline 2000.0 & 500.0 & 0.1 & 1 & 0.2 & 0.075 & 0.04 & 0.2 \\
\hline \hline
\end{tabular}

Table 1: Physical and ionic parameters. 


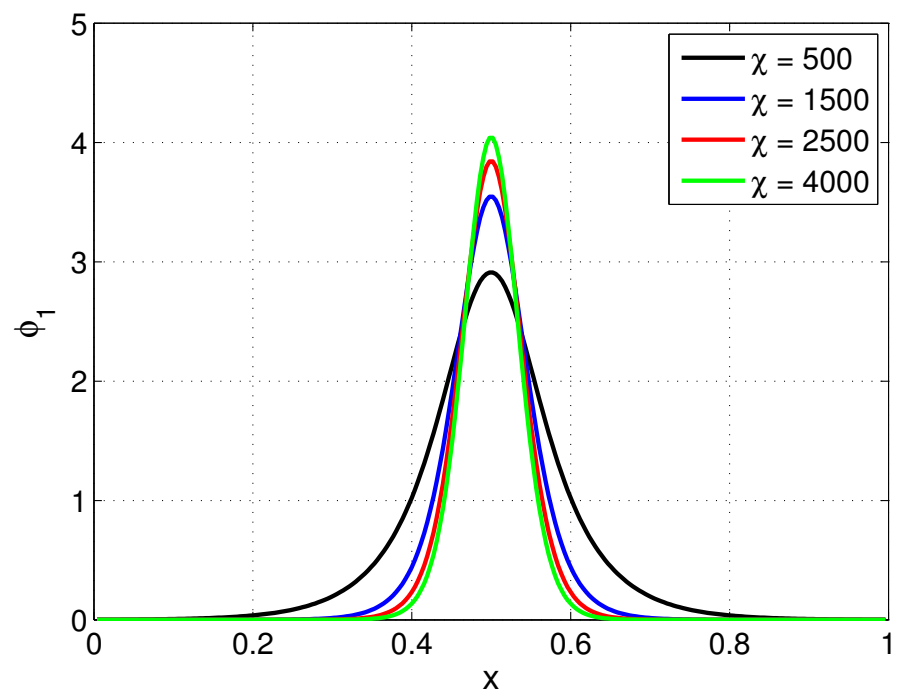

(a) First mode against $\chi$.

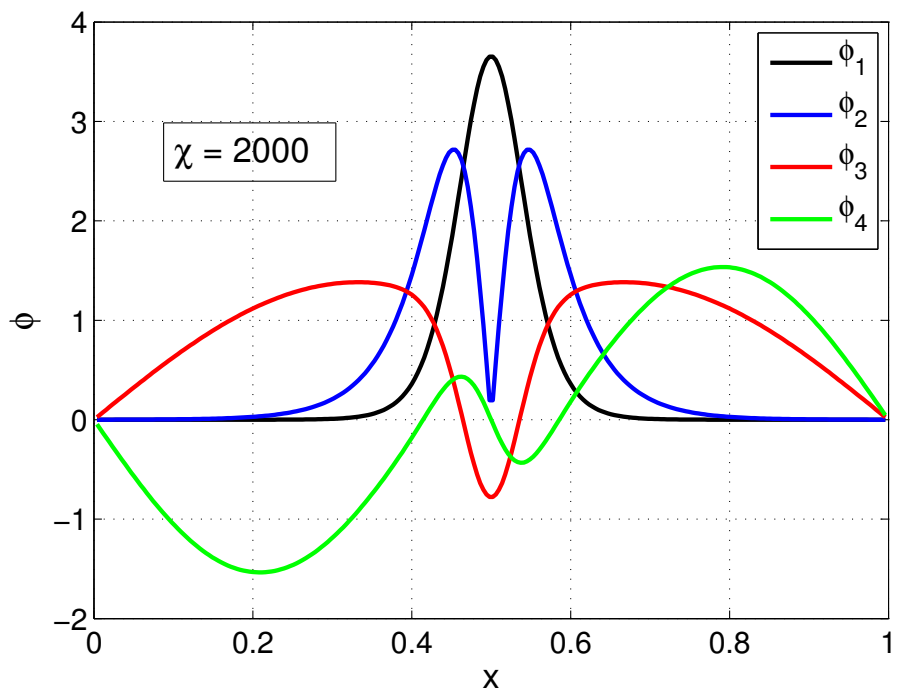

(b) The first modes when $\chi=2000$, mode 1 and 2 belong to the negative spectrum, while 3 and 4 to the positive one.

Figure 2: Eigenfunctions behavior. 
In order to build the initial time ALP basis $\Phi^{(0)}$ we consider the solution $v_{m}$ of the FEM simulation after $5 \mathrm{~ms}$. The basis is computed solving equation (30) with $\chi=15$. POD basis is generated by considering snapshots from the FEM simulation: 100 snapshots are retained with a sampling time of $0.5 \mathrm{~ms}$. The POD model is built by Galerkin projection.

Let us compare FEM and ROM solutions for ROM dimension space $N_{M}=25$. We observe good agreement between FEM solution and POD one (see first and last columns of Figure 3). ALP solution is in good accordance with FEM one, too (see first and second columns of Figure 3).

Then, we compare the relative $L^{2}\left(\mathbb{R}^{\mathcal{N}}\right)$ norm errors between FEM solution and ROM ones, computed by

$$
\operatorname{error}^{2}\left(t^{n}\right)=\frac{\left|u_{F E M}^{(n)}-u_{R O M}^{(n)}\right|^{2}}{\left|u_{F E M}^{(n)}\right|^{2}}
$$

for each time iteration $t^{n}, n \geq 0$, where $|\cdot|^{2}$ denotes the discrete norm $|v|^{2}=\sum_{i} v_{i}^{2}$, and $u_{F E M}^{(n)}, u_{R O M}^{(n)}$, represents the FEM, respectively ROM, solution at time $t^{n}$ in the high dimensional space $R^{\mathcal{N}}$. We compute the errors varying the dimension of ALP space: $N_{M}=15,20,25,30$. In Figure 4 we observe the decreasing values of ALP error with respect to the space dimension. In particular, each continuous curve (ALP errors) has a relative minimum after $20 \mathrm{~ms}$, i.e. when the depolarization front is already gone and the repolarization has not started yet. The relative error increases for any value of $N_{M}$ after 30 to $35 \mathrm{~ms}$, which represents the exit of the repolarization from the domain, this is due to some boundary effects caused by the low dimensional approximation. We observe the same behavior for the POD curve, too (dotted line). Finally, we remark that ALP relative error with $25-30$ modes is less than 10 percent during the whole simulation. We point out that the POD method gives an optimal solution because of the basis is build ad hoc. As we observe in next sections, the POD basis is not as efficient for any perturbation of the signal.

Concerning the computational costs of the algorithm, the gain of the ALP method for the monodomain equations (3) with $N_{M}=25$ modes was investigated. When a full reconstruction in the physical space is performed, the gain is about $20 \%$ compared to the FEM, including all the stages of the method. This speedup is clearly insufficient, and its improvement is the object of an on-going work. However, it is worth noticing that, in many applications, the reconstruction in the FE space is not necessary. As will be shown in Section 4.6, this is for example the case when only an output of interest depending linearly on the solution is needed. In this case, the update of the basis can be avoided and the ALP method has a computational cost which is one order of magnitude smaller than that of FEM (a speedup of about 8 was observed in our simulations). 

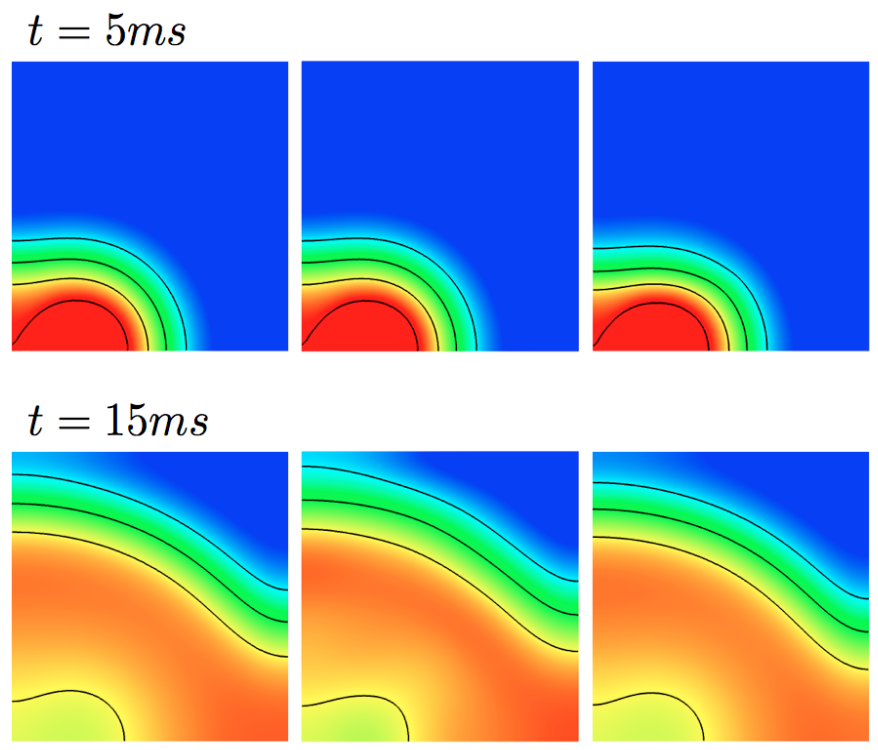

$$
t=20 \mathrm{~ms}
$$
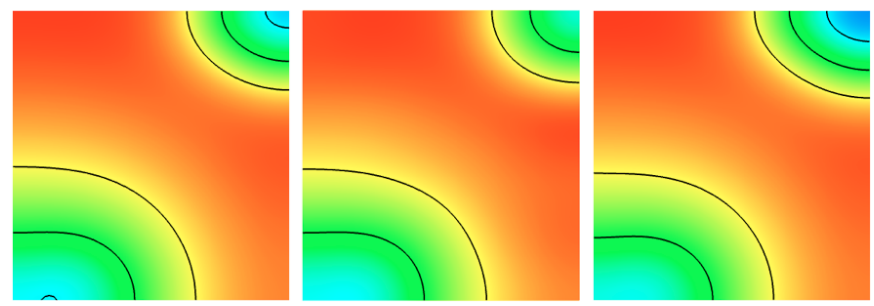

$$
t=25 \mathrm{~ms}
$$
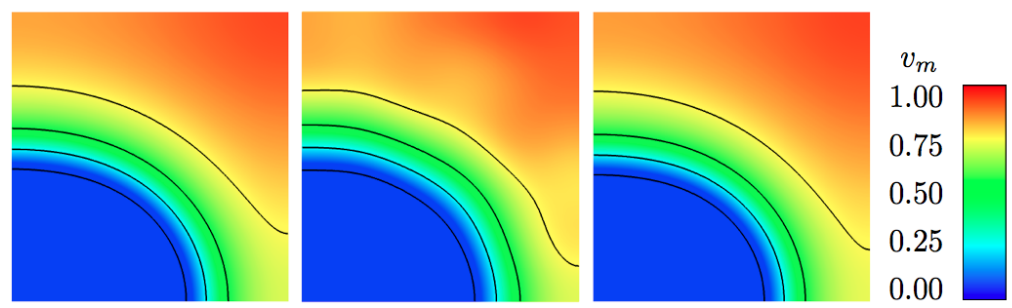

Figure 3: Comparison between FEM (left column), ALP $\left(\chi=15, N_{M}=25\right)$ in the center and POD $\left(N_{M}=25\right)$ on the right for the homogeneous tissue test case (section 4.3). Four different times are considered, namely $t=5,15,20,25 \mathrm{~ms}$. 


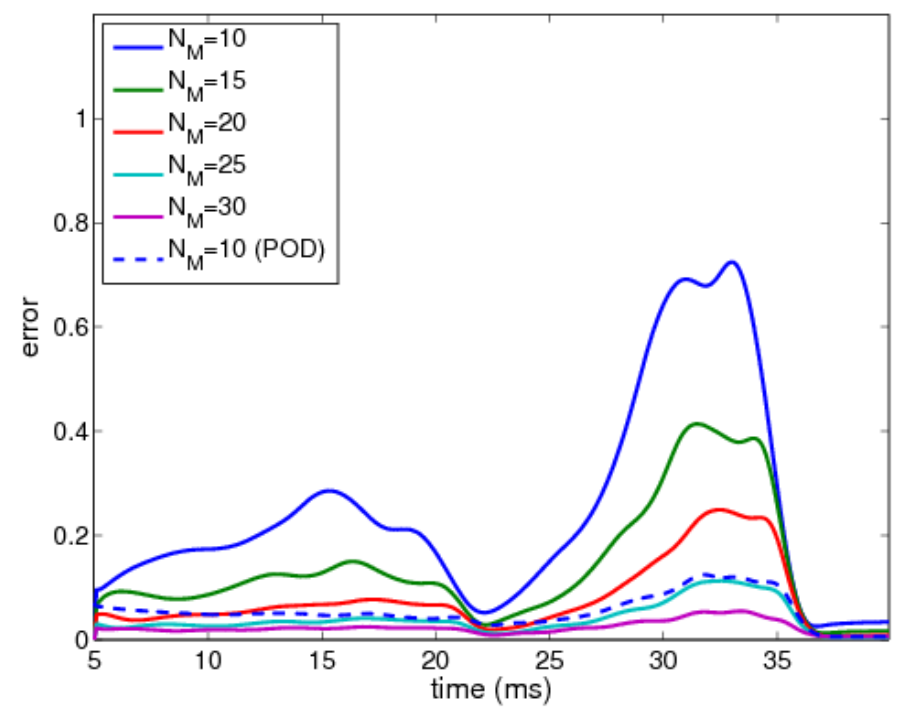

Figure 4: Relative errors in $L^{2}$ norm for the homogeneous tissue test case (section 4.3), varying the number of modes used, for ALP method (continuous lines, $\chi=15$ ) and POD (dotted line).

\subsection{Heterogeneous ionic parameters}

A test case with heterogeneous ionic parameter $s$ is presented in this section, which is challenging from a ROM point of view. Indeed, $s(x)$ is a function of the space, it is a distributed parameter that belongs to a high dimensional (infinite dimensional) space. For the reduced-order techniques that rely on the pre-computation of solutions, this would increase in a significant way the database dimension and the cost of the offline phase. In particular, for the test case considered, the expression of $s(x)$ reads:

$$
\begin{array}{r}
s(\mathbf{x})=s_{0} \cdot \mathbb{1}_{\Omega / \Omega_{s}}+s_{1}(\mathbf{x}) \cdot \mathbb{1}_{\Omega_{s}}, \\
\Omega_{s}=\{(x, y) \mid(0 \leq x \leq 0.5),(0.25 \leq y \leq 0.75)\} \\
s_{1}(\mathbf{x})=s_{0} \frac{36 y-7}{20} .
\end{array}
$$

The value of the parameter $s_{0}$ is reported in Table 1 . There is a square subdomain in which the ionic parameter $s$ is modified, its value is linear with respect to $y$ : being only $1 / 10$ with respect to the nominal one in the inferior border $(y=0.25)$ and $s_{0}$ in the upper boundary $(y=0.75)$. This can be seen as a schematic representation of an obstacle for the depolarization waves.

The ALP method was applied to this scenario. It is worth noting that the initial condition for this simulation is the same as for the homogeneous test case, so that the initial modes set is exactly the same. The ALP ROM was integrated, taking $\chi=15$ and 


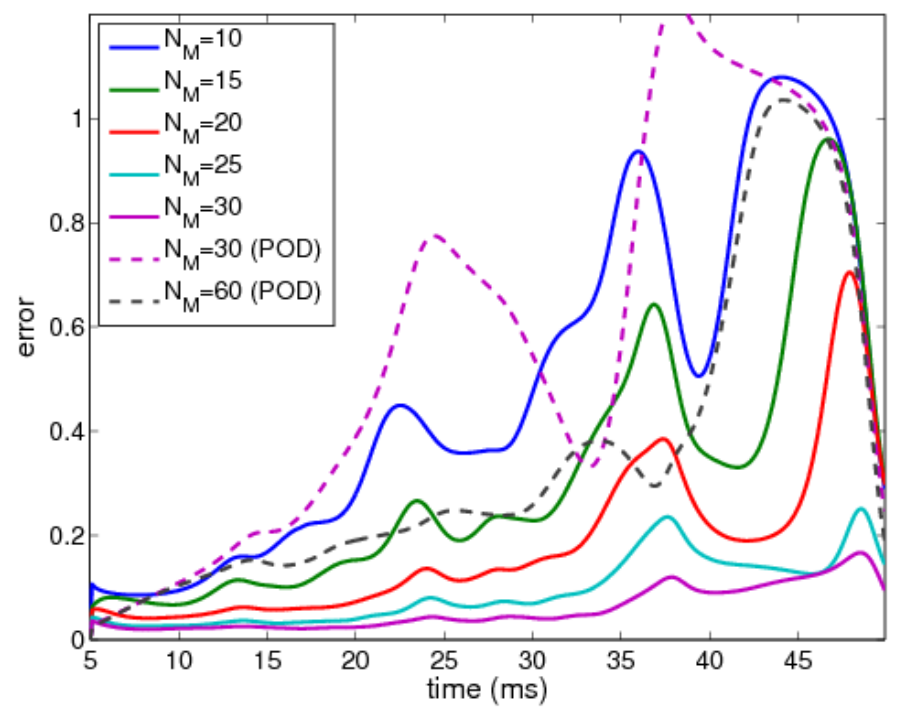

Figure 5: Relative errors in $L^{2}$ norm for the heterogeneous ionic parameter test case (section 4.4), varying the number of modes used, for ALP method (continuous lines, $\chi=15)$ and POD (dotted line).

a time step $\delta t=0.01 \mathrm{~ms}$. In Figure 5 the $L^{2}$ relative error between the FEM solution and the reconstruction of the ALP-ROM one is shown as function of time, varying the number of modes used. The errors are larger with respect to those observed for the homogeneous test case presented in the previous section. This can be explained by considering that in presence of an obstacle the dynamics is complex and a larger number of modes is needed to render it. As expected the error decreases when the number of modes is increased and it is less than $10 \%$ when $N_{M}=30$ modes are used. The error globally increases in time, but it is not monotonically increasing. The peaks observed corresponds to boundary interactions of the depolarization wave. The dashed lines are the relative errors of the POD reduced-order model when $N_{M}^{(P O D)}=30$ and $N_{M}^{(P O D)}=60$. The error has peaks larger than $100 \%$ in both cases, and for $N_{M}^{(P O D)}=60$ it is overall comparable to ALP when $N_{M}=15$.

A qualitative comparison between ALP and POD solutions is proposed in Figure 6, at four different instants: $t=15,20,25,30 \mathrm{~ms}$. The FEM solution is represented on the left column, ALP is in the center and POD on the right. The POD model is not accurate enough out of database and it is not able to account for the dynamics in the presence of an obstacle, if this has not been taken into account in the database. The POD modes number has to be increased up to $N_{M}^{(P O D)}=60$ in order to start having a realistic behavior. On the contrary, ALP performance is remarkable, all the features of the solution are represented. The errors mainly concern the front shape (which is often 

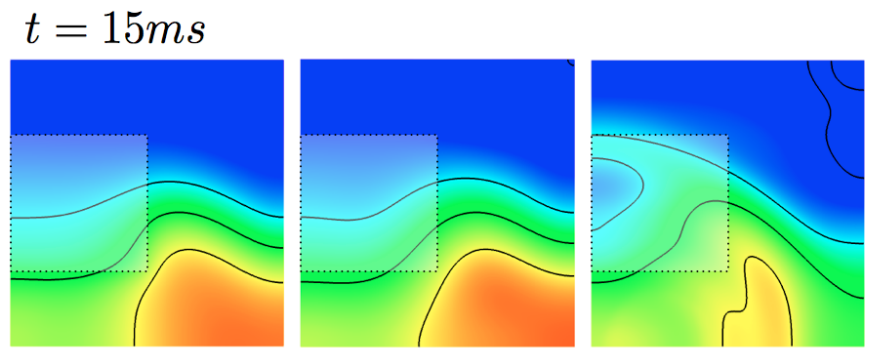

$$
t=20 m s
$$
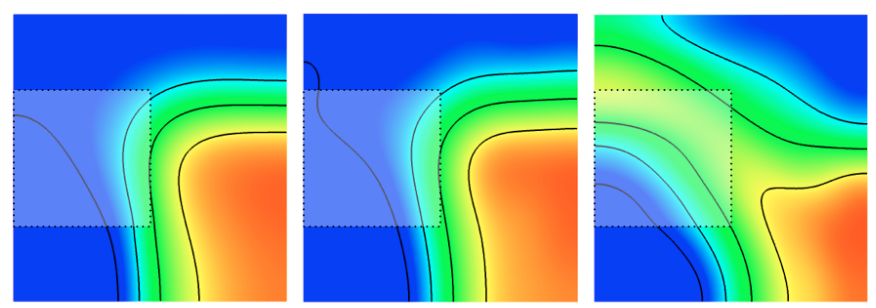

$$
t=25 \mathrm{~ms}
$$
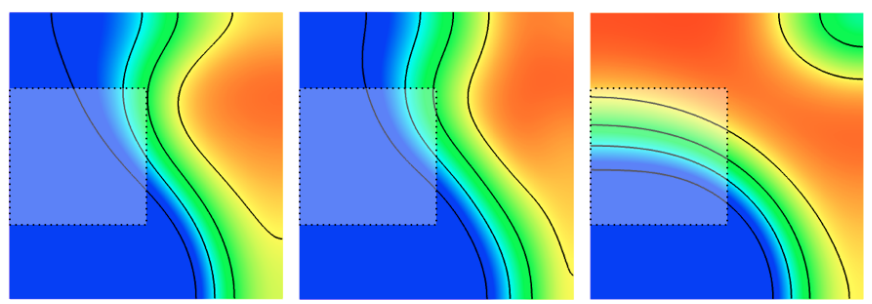

$$
t=30 \mathrm{~ms}
$$
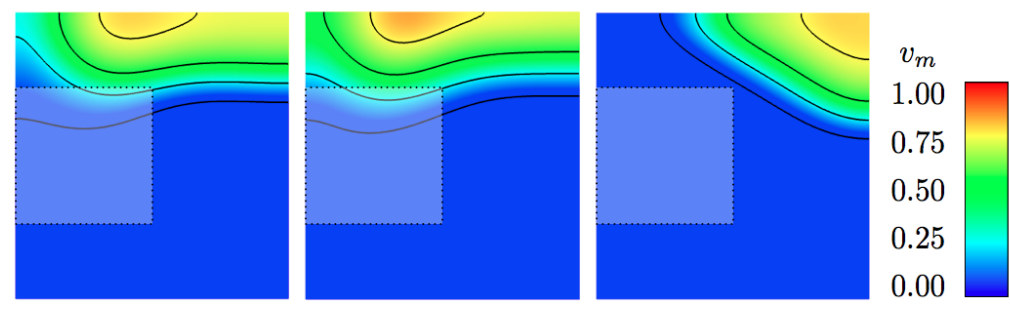

Figure 6: Comparison between FEM (left column), ALP $\left(\chi=15, N_{M}=25\right)$ in the center and POD $\left(N_{M}=25\right)$ on the right for the heterogeneous parameter test case (section 4.4). Four different times are considered, namely $t=15,20,25,30 \mathrm{~ms}$. 

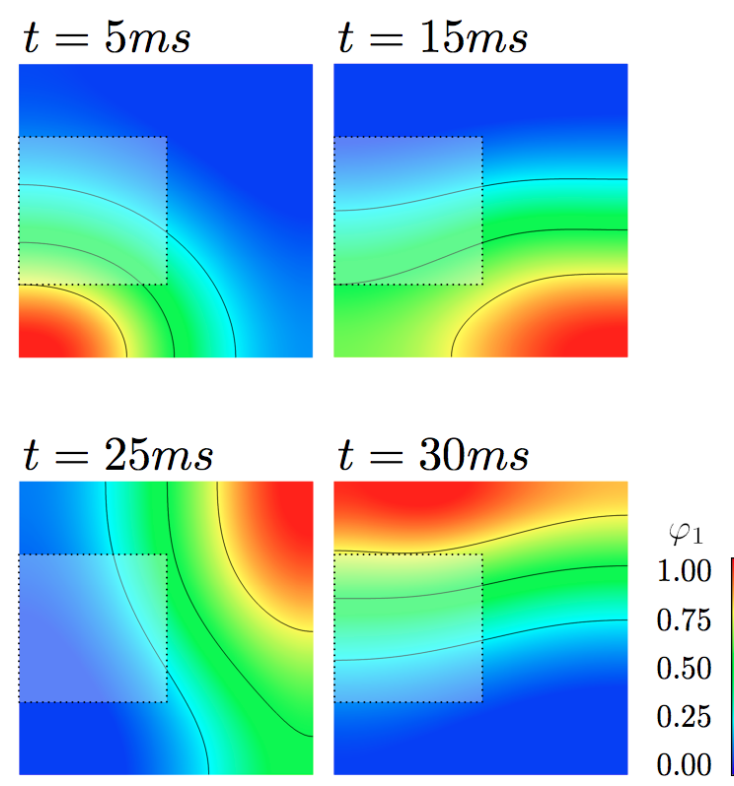

Figure 7: Evolution of the first mode for ALP-ROM when heterogeneous parameter test case (section 4.4) is integrated, at four different times: $t=5,15,25,30 \mathrm{~ms}$.

less sharp than the FEM one) and the boundary interactions.

In Figure 7 the time evolution of the first Schrödinger mode is considered, at four different times, $t=5,10,25,30 \mathrm{~ms}$. The mode basically evolves as the solution does. Indeed, the modification in the ionic parameter $s(x)$ makes the modal set evolve in order to match the dynamics of the system.

\subsection{Source terms}

In this section space-time sources are considered. The synthetic test presented hereafter is a schematized example of a realistic ectopic pacemaker. The proposed test case is as follows. The wave starts from the same initial condition as for the previous test cases. At $t=60 \mathrm{~ms}$ a source term is applied of the form:

$$
\begin{aligned}
& I_{a p p}(\mathbf{x}, t)=[H(t-60)-H(t-65)] z(\mathbf{x}), \\
& z(\mathbf{x})=0.04 \cdot \mathbb{1}_{\Omega_{c}},
\end{aligned}
$$

where $H$ denotes the heaviside function and

$$
\Omega_{c}=\left\{(x, y) \text { s.t. }\|(x, y-0.75)\|^{2} \leq 0.5\right\} .
$$

This is a challenging test case from a model-reduction point of view. Indeed, for a method relying on a database construction, a large number of stimulation locations and times should be pre-computed and the ROM usually performs poorly out-of-database. 


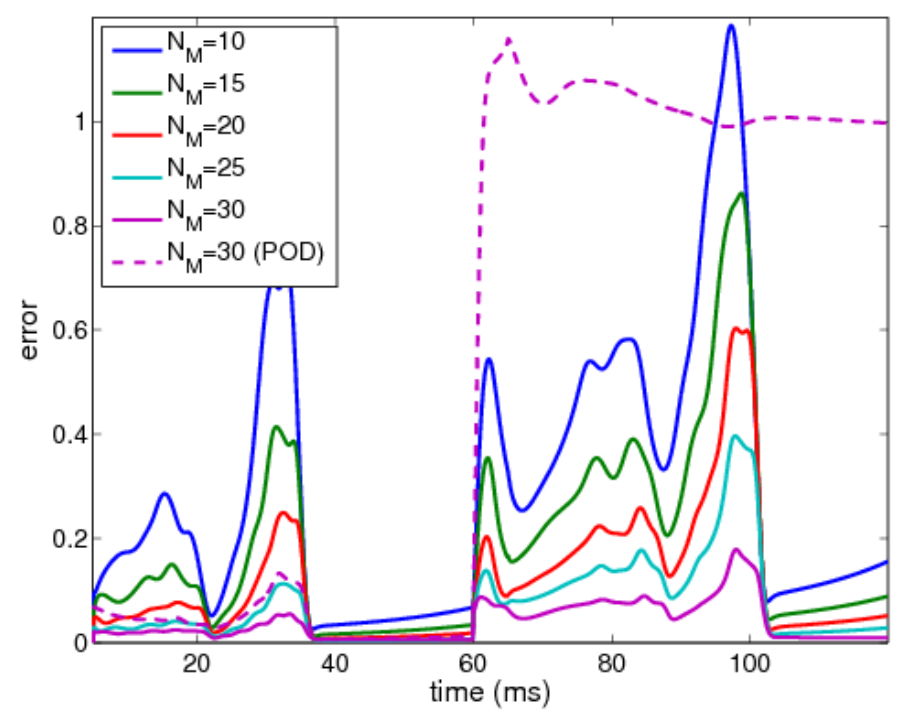

Figure 8: Relative error in $L^{2}$ norm for $\operatorname{ALP}(\chi=25)$ and POD with $N_{M}=30$ for the distributed source test case (see section 4.5).

The test was performed by varying the number of modes used. The initial condition for the modal set was extracted by taking $\chi=25$.

The relative error in $L^{2}$ norm was computed (see Figure 8). For $t<60 \mathrm{~ms}$ the curve is the same as for the homogeneous tissue test case presented in section 4.3. Let us comment the behavior of the scheme for $t>60 \mathrm{~ms}$. The error increases when the current is applied. After a transient phase the modal set adapts and the scheme is able to reproduce the dynamics of the system. As for the previous test cases, the error decreases as the number of modes is increased. The error is in general higher with respect to what observed in the homogeneous test case, with the same number of modes. This is another case for which the POD performances are poor. Indeed, when the sources are not precomputed the POD is not able to reproduce the correct results even with a high number of modes (see Figure 9). On the contrary, the ALP method errors are comparable to those obtained in the homogeneous test case (section 4.3).

In Figure 9, the FEM solution (left column) was compared to the solutions obtained with ALP (center column) and POD (right column) when $N_{M}=25$. Four different times are considered, namely $t=65,80,85,90 \mathrm{~ms}$. Between $t=60 \mathrm{~ms}$ and $t=65 \mathrm{~ms}$ the current source (dash-circle in the first row of the plots) has been applied. Its effect is not represented in the POD modes space since the snapshots were computed without it. Hence, at subsequent times, the polarization cannot propagate and the POD model is not able to provide the solution of the system. In spite of some errors in the position and the shape of the front, ALP is able to account for the wave propagation induced by the 

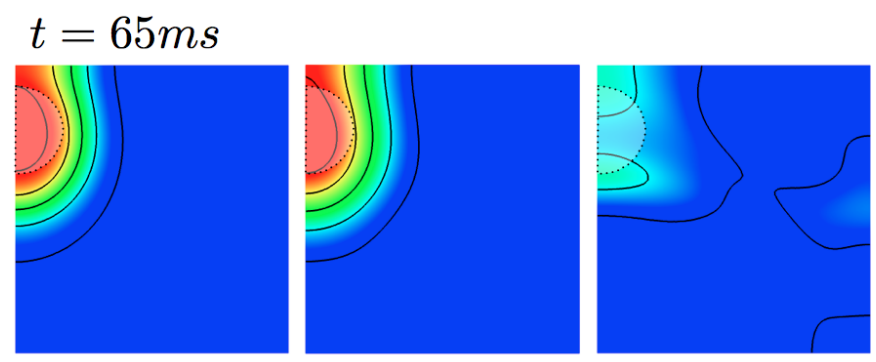

\section{$t=80 m s$}
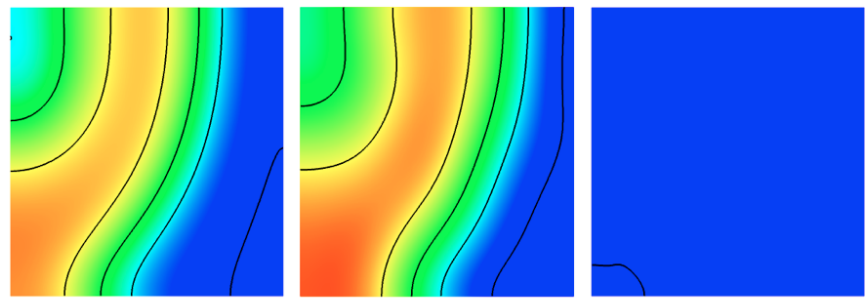

\section{$t=85 \mathrm{~ms}$}
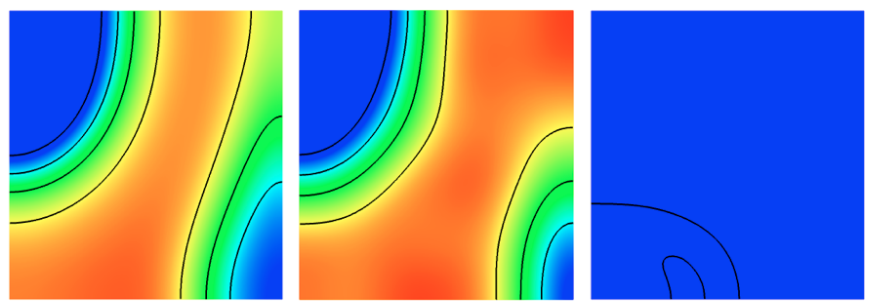

\section{$t=90 \mathrm{~ms}$}
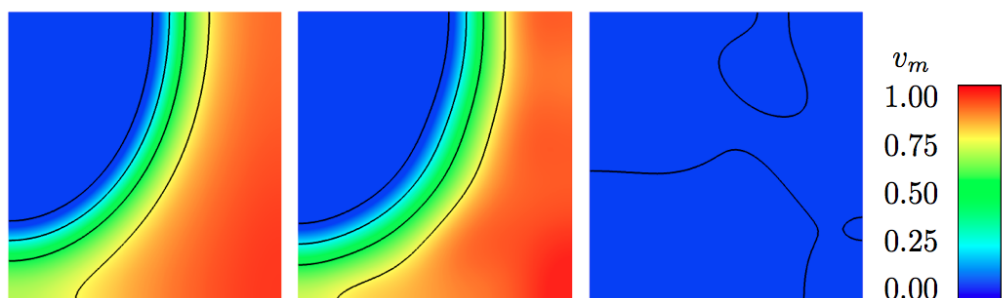

Figure 9: Comparison between FEM (left column), ALP $\left(\chi=15, N_{M}=25\right)$ in the center and POD $\left(N_{M}=25\right)$ on the right for the source test case (section 4.5). Four different times are considered, namely $t=65,80,85,90 \mathrm{~ms}$. For the first part of the simulation, $t<60 \mathrm{~ms}$, see Figure 3 (test case of section 4.3). 
source.

\subsection{Pseudo-electrocardiograms}

We are now interested in an application related to electrocardiograms (ECG) computation. ECG represents an convenient and efficient medical test to control the heart behavior, it consists in measuring the electrical potential onto 9 skin points. From a mathematical point of view, the bidomain equations (1) are coupled to a diffusion problem in the torso:

$$
\operatorname{div}\left(\sigma_{T} \nabla u_{T}\right)=0, \text { in } \Omega_{T},
$$

where $u_{T}$ represents the torso electrical potential and $\sigma_{T}$ the torso conductivity, an heterogeneous parameter which takes into account for instance bones and lungs conductivities. Equations (65) can be coupled to (1) by imposing the continuity of the extracellular potential and current. In this study, we consider a weak coupling between heart and torso potential, i.e. only the potential continuity is imposed. Then the boundary conditions for $(65)$ are

$$
\begin{aligned}
u_{T} & =u_{E}, & & \text { on } \Sigma \\
\sigma_{T} \nabla u_{T} \cdot n & =0, & & \text { on } \partial \Omega_{T}^{\text {ext }}
\end{aligned}
$$

where $\Sigma=\partial \Omega_{H}$ represents the external boundary of the heart domain and $\partial \Omega_{T}^{\text {ext }}$ indicates the external boundary of the torso domain $\Omega_{T}$.

Since we now consider the bidomain equations, we have to treat the extracellular variable. In section 3.3 the ionic variable $w$ was approximated in the same reduced order space as the transmembrane potential. We do the same for the extracellular potential $u_{E}$ and write

$$
\hat{u}_{E}=\sum_{i=1}^{N_{M}} \xi_{i}(t) \varphi_{i}(x, t),
$$

where $\hat{u}_{E}$ is the low dimensional approximation of $u_{E}, \xi_{i}(t)$ is the representation of $\hat{u}_{E}$ in the reduced order space $\Phi$.

We denote by $y=\left(u_{T}\left(x_{1}^{E C G}\right), \ldots, u_{T}\left(x_{N_{E C G}^{E C G}}^{E C)}\right)\right.$ the values of $u_{T}$ at $N_{E C G}$ locations on the boundary of the torso. The measurement $y$ is the output of interest of this problem. It is related to $u_{E}$ by the relation

$$
y=S \Pi u
$$

where $\Pi: \mathbb{R}^{\mathcal{N}} \mapsto \mathbb{R}^{N_{\Sigma}}$ denotes the boundary restriction operator, $N_{\Sigma}$ is the number of vertices on heart/torso interface, $S \in \mathbb{R}^{N_{E C G} \times N_{\Sigma}}$ denotes the heart-torso transfer matrix, and $u \in \mathbb{R}^{\mathcal{N}}$ denotes the degrees of freedom of the extracellular potential $u_{E}$ in the finite element space. If we consider the representation of $u$ in the reduced-order space, $u=\Phi \xi$, then the ECG measurements can be written as

$$
y=X \xi
$$




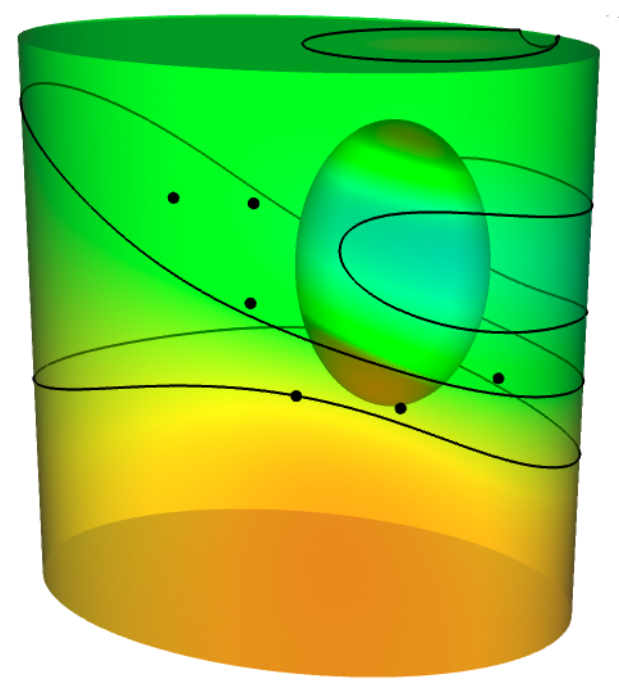

Figure 10: FEM heart-torso equations solution at time $t=25 \mathrm{~ms}$.

where

$$
X:=S \Pi \Phi \in \mathbb{R}^{N_{E C G} \times N_{M}}
$$

is a very small matrix. This linear operator $X$ commutes with time, so we can apply the same technique as in section 3.1 in order to compute its time derivative:

$$
\dot{X}_{i j}=\sum_{l=1}^{N_{M}} X_{i l} M_{l j} .
$$

So, the ECG measurement $y$ is computed at each time iteration using the low dimensional vector $\xi$ and the matrix $X$ whose update is easily computed using (71), without any reconstruction of the solution in the finite element space.

For the numerical simulations, we consider an ellipsoid, representing the heart, embedded in a cylinder, represented the torso. The mesh of the ellipsoid has 22.140 vertices, while the external one has 338.920 vertices, of which 7.572 are on the interface $\Sigma$ between the two domains.

We first solve the bidomain equations (1) in the ellipsoid using FEM and a second order Backward Difference method to integrate in time. The conductivity tensors $\sigma_{I}$ and $\sigma_{E}$ are considered isotropic, their values are $\sigma_{I}=1, \sigma_{E}=4$. Then, we solve the torso diffusion problem (65) with boundary conditions (66) where the extracellular potential is taken from the bidomain solution in the ellipsoid. A snapshot of the solution at time $t=20 \mathrm{~ms}$ is shown in Figure 10. In particular we are interested in the torso potential measured by 6 electrodes positioned in the black points of Figure 10.

Concerning the ALP resolution, we compute the initial basis $\Phi$ using the operator $\mathcal{L}_{\chi}$ defined in (44) where $u$ is the FEM solution at time $t=5 \mathrm{~ms}$ and $\chi=50$. We solve then 

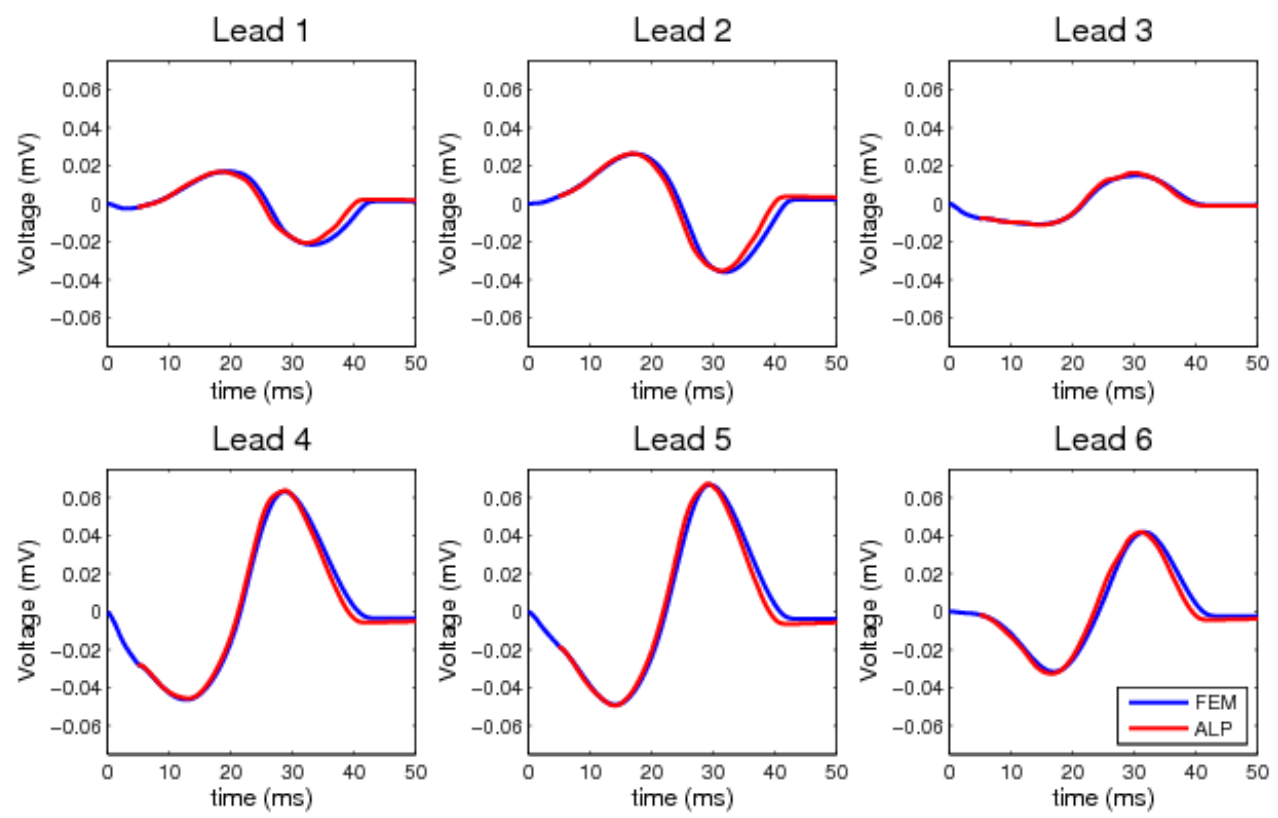

Figure 11: Comparison between FEM electrodes measures (blue line) and ALP ones (red line) for $\chi=50, N_{M}=60$.

equations (51) and we compute by equation (69) the 6 torso measures we are interested in. We remark that, as the only output we want to show are the 6 electrodes measures, no reconstruction in the FEM space is needed.

Figure 11 compares FEM measures with ALP results run for $N_{M}=60$. We observe that ALP curves (red lines) are in good accordance with FEM ones (blue lines), the signal is well reproduced on every leads.

\section{Conclusions and perspectives}

The ALP method can be seen as a technique to propagate a basis in a way related to the dynamics of the solution. In this paper, ALP was applied to the simulation of highdimensional parametric systems arising in cardiac electrophysiology. The approach was assessed in a wide range of different situations, including 2D and 3D complex configurations. The main advantage with respect to standard approaches consists in the fact that no database of solutions has to be built to define the reduced-order model.

Several perspectives are in order. The first one is the choice of the basis to propagate, that is, the choice of the operator $\mathcal{L}_{\chi}$. To derive the method, $\mathcal{L}_{\chi}$ has to be selfadjoint, with a compact inverse and linear in $u$. In this paper, we chose a specific expression for $\mathcal{L}_{\chi}$ but many others are possible and would lead to different results and performances. This 
aspect deserves further investigation. Another important perspective is the possibility to deal with nonpolynomial nonlinearities. To do so, we are currently working on an extension of ALP using the concepts of empirical interpolation, as introduced in $[2,6]$. This approach will allow us to consider more realistic models of electrophysiology, including physiological ionic models. The $N_{M}^{3}$ complexity, due to the propagation of tensor $T$, is another limitation of the current version of ALP. It is expected that the use of empirical interpolation will also overcome this issue. Last but not least, the analysis of the stability and accuracy of the method is still missing and deserves to be addressed in future works.

\section{References}

[1] David Amsallem and Charbel Farhat. Interpolation method for adapting reducedorder models and application to aeroelasticity. AIAA Journal, 46(7):1803-1813, 2008.

[2] Maxime Barrault, Yvon Maday, Ngoc Cuong Nguyen, and Anthony T Patera. An empirical interpolationmethod: application to efficient reduced-basis discretization of partial differential equations. Comptes Rendus Mathematique, 339(9):667-672, 2004.

[3] M. Boulakia, S. Cazeau, M.A. Fernández, J.-F. Gerbeau, and N. Zemzemi. Mathematical modeling of electrocardiograms: a numerical study. Annals of Biomedical Engineering, 38(3):1071-1097, 2010.

[4] Muriel Boulakia, Elisa Schenone, and Jean-Frédéric Gerbeau. Reduced-order modeling for cardiac electrophysiology. Application to parameter identification. International Journal for Numerical Methods in Biomedical Engineering, 28:727-744, 2012. RR-7811 RR-7811.

[5] K. Carlberg, C. Bou-Mosleh, and C. Farhat. Efficient non-linear model reduction via a least-squares petrov-galerkin projection and compressive tensor approximations. International Journal for Numerical Methods in Engineering, 86(2):155-181, 2011.

[6] S. Chaturantabut and D. Sorensen. Nonlinear model reduction via discrete empirical interpolation. SIAM Journal of Scientific Computing, 32(5):2737-2764, 2010.

[7] M. Cheng, T.Y. Hou, and Z. Zhang. A dynamically bi-orthogonal method for time-dependent stochastic partial differential equations i: Derivation and algorithms. Journal of Computational Physics, 242:843-868, 2013.

[8] M. Cheng, T.Y. Hou, and Z. Zhang. A dynamically bi-orthogonal method for timedependent stochastic partial differential equations ii: Adaptivity and generalizations. Journal of Computational Physics, 242:753-776, 2013. 
[9] P Colli-Franzone, LF Pavarino, and S Scacchi. Exploring anodal and cathodal make and break cardiac excitation mechanisms in a $3 \mathrm{~d}$ anisotropic bidomain model. Mathematical Biosciences, 230(2):96-114, 2011.

[10] P. Colli Franzone, L.F. Pavarino, and B. Taccardi. Simulating patterns of excitation, repolarization and action potential duration with cardiac Bidomain and Monodomain models. Mathematical Biosciences, 197(1):35-66, 2005.

[11] R. Fitzhugh. Impulses and physiological states in theoretical models of nerve membrane. Biophysical Journal, 1:445-465, 1961.

[12] Jean-Frédéric Gerbeau and Damiano Lombardi. Approximated Lax Pairs for the Reduced Order Integration of Nonlinear Evolution Equations. Research Report RR8454, INRIA, January 2014.

[13] P. Holmes, J.L. Lumley, and G. Berkooz. Turbulence, coherent structures, dynamical systems and symmetry. Cambridge University Press, 1st edition, 1996.

[14] O. Koch and C. Lubich. Dynamical low rank approximation. SIAM Journal of Matrix Analysis and Applications, 29:435-454, 2007.

[15] K Kunisch, S Volkwein, and L Xie. Hjb-pod-based feedback design for the optimal control of evolution problems. SIAM Journal on Applied Dynamical Systems, 3(4):701-722, 2004.

[16] P. D. Lax. Integrals of nonlinear equations of evolution and solitary waves. Communications on Pure and Applied Mathematics, 21:467-490, 1968.

[17] J.S. Nagumo, S. Arimoto, and S. Yoshizawa. An active pulse transmission line simulating nerve axon. Proceedings of the IRE, 50(10):2061-2070, 1962.

[18] M. Pennacchio, G. Savaré, and P. Colli Franzone. Multiscale modeling for the bioelectric activity of the heart. SIAM Journal on Mathematical Analysis, 37(4):1333-1370, 2005.

[19] G. Rozza, D.B.P. Huynh, and A.T. Patera. Reduced basis approximation and a posteriori error estimation for affinely parametrized elliptic coercive partial differential equations. Archives of Computational Methods in Engineering, 15(3):1-47, 2007.

[20] D. Ryckelynck, F. Vincent, and S. Cantournet. Multidimensional a priori hyperreduction of mechanical models involving internal variables. Computer Methods in Applied Mechanics and Engineering, 225:28-43, 2012.

[21] F.B. Sachse. Computational Cardiology: Modeling of Anatomy, Electrophysiology and Mechanics. Springer-Verlag, 2004. 
[22] T.P. Sapsis and Lermusiaux P.F.J. Dynamically orthogonal field equations for continuous stochastic dynamical systems. Physica D, 238:2347-2360, 2009.

[23] L. Sirovich. Low dimensional description of complicated phenomena. Contemporary Mathematics, 99:277-305, 1989.

[24] D.D. Streeter. Gross morphology and fiber geometry of the heart. Handbook Physiology, The cardiovascular system, 1:61-112, 1979.

[25] J. Sundnes, G.T. Lines, X. Cai, B.F. Nielsen, K.A. Mardal, and A. Tveito. Computing the Electrical Activity in the Heart, volume 1 of Monographs in Computational Science and Engineering. Springer-Verlag, 2006. 